\title{
Tinea capitis in primary and middle school students in South-East of Iran
}

\author{
Ebrahimzadeh Adel $^{1,2, ~ *, ~ H a s h a m i ~ S h a h r i ~ S a e e d ~ M o h a m m a d ~}{ }^{2,3}$, Fazaeli Asghar ${ }^{2,4}$ \\ ${ }^{1}$ Department of Parasitology and Mycology, Medical School, Zahedan University of Medical Sciences, Zahedan, Iran \\ ${ }^{2}$ Zahedan Research Center for Infectious Diseases \& Tropical Medicine, Zahedan, Iran \\ ${ }^{3}$ Bo-Ali Hospital, Zahedan University of Medical Sciences, Zahedan, Iran \\ ${ }^{4}$ Department of Parasitology and Mycology, Medical School, Zanjan University of Medical Sciences, Zanjan, Iran
}

\section{Email address:}

adel1336@yahoo.com (Ebrahimzadeh A.)

\section{To cite this article:}

Ebrahimzadeh Adel, Hashami Shahri Saeed Mohammad, Fazaeli Asghar. Tinea Capitis in Primary and Middle School Students in SouthEast of Iran. Science Journal of Public Health. Vol. 2, No. 3, 2014, pp. 205-208. doi: 10.11648/j.sjph.20140203.21

\begin{abstract}
Tinea capitis is a common infection among school children, still raising public health importance in many countries. There are unpublished reports of head fungal infections, particularly Tinea capitis, in school children of Sistan and Baluchestan province, at South-East of Iran. However, the reports are only based on the clinical evidences and there is no information about the prevalence and the agents of the infection. The present study was designed to determine the prevalence of Tinea capitis based on demographic information as well as laboratory determination of the causative fungal species in primary and middle school students of seven districts in Sistan and Baluchestan in 2011. During this crosssectional study, 2060 children from urban and rural primary and middle schools were randomly selected and subjected to the survey by informed consents. Their demographic information was recorded in questionnaire and specimens from suspicious students were taken by scraping the scalp and removing hair stubs. Microscopic examination and culture and subculture of the specimens were performed using Sabouraud's Dextrose Agar (S) and S + Chloramphenicol + Cyclohexamide (SCC) media and slide culture for identification of fungal agents. The surveyed children contained $56.7 \%$ female and $43.3 \%$ male. A number of 110 students (5.8\%) were suspected to have Tinea capitis. Thirty-seven cases of Tinea capitis were confirmed by demonstration of dermatophytes spores or mycelium in direct smears, consisting of 25 (22.73\%) ectothrix, $8(7.27 \%)$ endothrix and 4 cases (3.63\%) of favus. The dermatophytes isolated by in in vitro cultures, included 17 anthropophilic, 9 zoophilic and 3 geophilic species. The other 7 isolates were only characterised as Trichophyton mentagrophytes but remained unidentified at the level of subspecies. The prevalence of Tinea capitis in the rural school students was significantly more than that in the urban students. The infection rate in male was also significantly more than that in female. The results implied that control program with concern to the sources of infections is of great importance. Health education and development of knowledge among school children are recommended to be taken into account. It was resulted that all three sources of dermatophyte infections are involved with the incidence of Tinea capitis in children at South-East of Iran, although the anthropophilic and zoophilic reservoirs are predominant.
\end{abstract}

Keywords: Tinea capitis, Dermatophyte, South-East, Iran, School

\section{Introduction}

The dermatophytic infection is considered a public health problem. Tinea capitis is the name used for infection of the scalp with dermartophytes that are common in children, and is less frequently seen in adults. The infection usually afflicts further children, aged between 3 to 7 years [1]. It is a fungal infection of the scalp hair follicles caused by different species of the genera Microsporum and Trichophyton. This infection may have a variety of clinical presentations such as inflammatory or non-inflammatory form and carrier state [2]. The non-inflammatory form may include gray-patch scaling, seborrheic dermatitis-like scale, hair thinning without significant scaling, and distinct patches of black-dot alopecia. Inflammatory Tinea capitis also may have multiple clinical forms, including localized pustules that are similar to impetigo and multiple widespread abscesses that mimic dissecting cellulitis. Carrier state may present as a minimal infection [3]. Because dermatophytes are not part of the normal flora of 
the scalp skin, it is important to understand the significance of their presence in different communities. Some asymptomatic Tinea capitis cases may go on to overt infection, whereas particular conditions may resolve the infection spontaneously. The carrier state is likely to be a reservoir of infection. The factors that determine the clinical appearance of childhood Tinea capitis, have not been investigated in great details, but stimulation of cellmediated immunity is widely accepted as a cause of inflammation [2]. This dermatophyte disease is considered a public health problem, particularly among school children in developing countries [3,4]. This condition is still under reported in Iran. We conducted a survey on primary and middle school students to determine the prevalence of Tinea capitis based on demographic information as well as laboratory determination of the causative fungi in seven districts of Sistan and Baluchestan province, situated in South-East of Iran in 2011.

\section{Materials and Methods}

The study was conducted in seven districts (Zahedan, Khash, Saravan, Iranshahr, Sarbaz, Nikshahr and Chabahar) of Sistan and Baluchestan province, Southeastern of Iran. A number of 2060 students from primary schools and middle schools, from both sexes, were randomly selected. Permission slips and questionnaires were sent home with the students two weeks before the scheduled school visit; those eligible had their permission forms returned by a parent or guardian, were enrolled in the study. The questionnaire requested information on basic demographic characteristics, clinical history, environmental exposures and behavioral characteristics. In addition, detailed questions regarding hair care and use of various hair products were included. A team, consisting of a dermatologist and laboratory technologists, visited each school during regular school hours. Students were examined in classroom or in the office of the school nurse. A Wood's lamp was used to detect evidence of fluorescent fungal elements. Each child's scalp was examined for scaling, crusting and alopecia. If such areas were evident, a sample was obtained by vigorously rubbing the affected scalp with a piece of sterile scalpel or scraping the scalp and removing hair stubs and then collecting in a sterile plate. If no clinical signs and symptoms of Tinea capitis were present, the scalp was sampled along the top of the head and along the neckline with sterile scalpel. They were labeled and transferred to the medical mycology laboratory at Zahedan Medical School. A direct smear was prepared from each sample, using lacto-phenol, followed by microscopic examination and a part of sample was cultured on Sabouraud's Dextrose Agar (S). After preliminary isolation of fungal agents, they were sub-cultured onto the SCC (S + Chloramphenicol + Cyclohexamide) for specific identification. Slide cultures (Riddle assay) were also used for identification of genus and species of the isolated dermatophytes [5]. The results were supplied by mail to the child's home so that the family doctor or clinic could provide treatment if needed. To preserve confidentiality, the individual results were not provided to the schools.

\section{Results}

A total of 2060 School children, 1145 (55.58\%) from primary schools and 915 (44.42) from middle schools were examined for any clinical manifestation of Tinea capitis. They included 892 (43.3\%) boys and 1168 (56.7\%) girls, aged between 7 to 14 years.

From the students subjected to observation, a number of $110(5.33 \%)$, including 83 primary students (7.2\%) and 27 middle school students $(2.9 \%)$ were suspected to have Tinea capitis, consisting of $96(10.7 \%)$ boys and $14(1.2 \%)$ girls. A proportion of $75.45 \%$ (83) of the suspected cases reported no symptoms. No cases exhibited kerion or severe inflammation on examination. However, a number of 587 $(28.5 \%)$ of the examined students, complained of scalp itching, 26 (1.3\%) had scaling, $232(12.7 \%)$ had some hair loss, $98(4.8 \%)$ had previously experienced Tinea capitis infection and $86(4.1 \%)$ had previous antifungal treatment with griseofulvine. Concerning the settlement areas, 904 of the participants were from urban schools with the prevalence of $4.3 \%$ (39 cases) of Tinea capitis and the remaining 1156 students were from rural schools with the prevalence of $6.1 \%$ (71 cases). In the rural schools, it was significantly more than that in the urban schools. Thirty-six out of 110 suspected Tinea capitis cases (33.63\%) were confirmed by demonstration of dermatophytes spores or mycelium in direct smears, identifying 24 cases $(22.73 \%)$ ectothrix, 8 cases $(7.27 \%)$ endothrix and 4 cases $(3.63 \%)$ of favus. The five students stated to have a pet at home, but no significant association was seen between Tinea capitis and pet keeping. The education level of parents, taking frequent showers at least twice a week, and using healthy water demonstrated to have role in preventing Tinea capitis. Thirty-six cases of dermatophytes were confirmed by in vitro cultures and led to the species identification; most common species was Trichophyton rubrum, followed by several other species as mentioned in Table 1.

Table 1. Dermatophyte fungal species isolated from Tinea capitis diagnosed among the school students in Sistan va Baluchestan province, Southeast of Iran (2007).

\begin{tabular}{lll}
\hline Dermatophytes Spp. & No & \% \\
\hline Trichophyton rubrum & 10 & 27.8 \\
Trichophyton mentagrophytes spp. & 7 & 19.5 \\
Trichophyton schoenleinii & 4 & 11.0 \\
Trichophyton verrucosum & 3 & 8.4 \\
Trichophyton violuceum & 2 & 5.5 \\
Microsporum canis & 6 & 16.7 \\
Microsporum gypseum & 3 & 8.4 \\
Microsporum audoinii & 1 & 2.7 \\
Total & 36 & 100 \\
\hline
\end{tabular}




\section{Discussion}

This study is the first documented survey in Tinea capitis based on both direct examination and culture in Iran. A relatively high prevalence (33.63\%) of dermatophytepositive scalp cultures was found among the suspected cases, with a high proportion of two genera of dematophyte fungi, Trichophyton spp (72.2\%) and Microsporum spp (27.8\%). The predominant species were T. rubrum, $T$. mentagrophytes and M. Canis.

Despite the differences of predominant species in the study area, this is higher than that reported earlier by Sharma et al, [6] who conducted a similar survey of 200 healthy children for scalp colonization and found that $4 \%$ of those cultured were positive for $T$. tonsurans. The differences in these incidences may reflect a difference in the study populations and methodologies [4]. It is not clear what the long-term clinical implications are for such asymptomatic carriage, because studies that have prospectively looked at long-term follow-up are limited. However, Williams et al, [7] from a similar study in a Philadelphia school, found that more than half of untreated asymptomatic carriers of $T$. tonsurans remained culture-positive after 2 months and suggested that these carriers play a role in the transmission of Tinea capitis within the school population. Unlike the findings of Sharma et al, [6] and an earlier study by Bronson et al, [8] that reported female African Americans to be more prone to scalp infection with dermatophyte-positive scalp cultures did not vary with gender, our study showed a higher incidence of the infection in boys in comparison with girls $(\mathrm{P}<0.05)$. Other studies have found that infection of the scalp with $T$. tonsurans occurs with equal frequency in two genders or slightly more commonly in boys. [9-11]. The present survey demonstrated a higher prevalence of Tinea capitis in the rural school students comparing to the urban students $(\mathrm{P}<0.05)$. The positive infection in primary schools was higher than in middle schools $(\mathrm{P}<0.05)$.

Our study exhibited that having a pet does not play a role in the prevalence of Tinea capitis. The findings showed that some factors such as parent's education, personal health, i.e. taking shower at least twice a week, and healthy water supply play important roles in prevention of the infection. Sharma et al, [6] suggested that the cultural practice of tight braiding and dressing of the hair with oils in young African American girls probably predisposes them to Tinea capitis. In contrast, our study showed that neither hair braiding nor the use of oils and cream rinses were associated with isolation of fungi from the scalp. However, sharing of hair items (eg., brushes, combs) or to transmission at barbershops and ownership of dogs or cats was not a predictor of colonization by dermatophytes fungi. This finding is not surprising, because T. rubrum is an anthropophilic fungus and has recently become the most common and widely distributed dermatophyte of man that spreads mainly from person to person, unlike the zoophilic M. canis. Studies by Babel and Baughman [12] have demonstrated that $T$. tonsurans, an anthropophilic fungus can be transmitted either directly or indirectly from child to child or from child to adult. Thus, once asymptomatic cases detected, it would be prudent to institute measures to avoid the occurrence of epidemics. Screening of adults in the households of infected children may limit reinfection. The predominance of T. rubum and T. mentagrophytes has a number of implications that can affect both patient management and laboratory testing. Infections caused by $M$. canis, M. audouinii and T. schoenleinii showed shine bluegreen fluoresce, but hairs infected by $T$. rubrum and $T$. mentagrophytes do not fluoresce under Wood's light, making detection of that organism much more difficult. Possible misdiagnosis of seborrheic dermatitis or dandruff may lead to the use of inappropriate therapy. Therefore, accurate diagnosis of Tinea capitis requires microscopic examination of hair and scalp scrapings as well as culture.

Many cases of non-inflammatory Tinea capitis observed in our study, might be due to the involvement of some particular dermatophyte species, an example of which is $T$. tonsurans that appears to exist in an asymptomatic state and never come to medical attention $[6,9]$. This scenario was clearly shown in our study population, as the vast majority of children in our survey were asymptomatic and thus might be considered as carriers. However, subjects with scaling were twice as likely to be culture-positive comparing to those showing no scaling. Our data analysis showed that the use of anti-dandruff shampoo was significantly higher in children who were culture-positive, indicating a misdiagnosis on the part of the child's caretaker and/or physician. Honig and Smith [13] suggest that what appears as seborrheic dermatitis or dandruff in a child should be suspect of fungal infection, and culture should be done in all cases. In fact, Tinea capitis should be suspected in all children who do not respond to antidandruff shampoo.

Our study demonstrated that the incidence of dermatophyte-positive scalp culture in children is increasing, the presence of scaling, and use of anti-dandruff shampoo being important predictors. These findings have important clinical implications for the diagnosis and management of Tinea capitis and raise further questions about its importance to public health. Will a greater awareness in physicians of this high prevalence reduce the confusion of Tinea capitis with dandruff in school-aged children should the high proportion of asymptomatic cases translate into routine mycological screening, and should all children with dermatophyte-positive scalp cultures be treated to eradicate a potential source of infection. These questions demonstrated the need for additional multi-center studies on a nationwide basis, with long-term follow-up of asymptomatic cases.

\section{Acknowledgements}

Our deep appreciation and thanks to the authorities of Health Service Center, Sistan and Baluchestan province and the Research Department at Zahedan University of Medical 
Sciences for their financial support to this study. The laboratory works of the study were carried out in Zahedan Research Center for Infectious Diseases \& Tropical Medicine.

\section{References}

[1] BE. Elewski, "Tinea capitis: a current perspective", J Am Acad Dermatol, 42: 1-20, 2000.

[2] BE. Elewski, "Topics in clinical dermatology: cutaneous fungal infections", New York: Igaku-Shoin, 1992.

[3] M. Ghannoum, "Tinea cpitis in Cleveland: Survey of elementary school students", J Am Acad Dermatol, 48:18993, 2003.

[4] N. Contet-Audonneau, P. Grosjean, L.R. Razankolona, T. Andriantsinjovina, R. Rapelanoro, "Tinea capitis in Madagascar: a survey in a primary school in Antsirabe, Ann Dermatol Venereol. 133(1): 22-5, 2006.

[5] J.W. Rippon, "Medical Mycology, the pathogenic fungi and the pathogenic actinomycetes, Third Edition", P: 154, 1988.

[6] V. Sharma, J. Hall, J. Knapp, S. Sarai, D. Galloway, D.
Babel," Scalpcolonization by Trichophyton tonsurans in an urban pediatric clinic", Arch Dermatol, 124:1511-3, 1988.

[7] JV. Williams, PJ. Honig,KJ. McGinley, JJ. Leyden, "Semiquantitativestudy of Tinea capitis and the asymptomatic carrier state in in-ner-city school children," Pediatrics, 96:265-7, 1995.

[8] DM. Bronson, DR. Desai, S. Barsky, SM. Foley, "An epidemic of infection with Trichophyton tonsurans revealed in a 20 year survey of fungal infections in Chicago", J Am Acad Dermatol, 8:322-30, 1983.

[9] E. Provost, "Tinea capitis in Charleston", SC. JAMA, 242:1765-7, 1979.

[10] JB. Howell, JW. Wilson, MR. Caro, "Tinea capitis caused by Trichophyton tonsurans (sulfureum or crateriforme)" Arch Dermatol, 65:194-205, 1952.

[11] JM. Beare,"Tinea capitis due to Trichophyton sulfureum", Br J Der-matol, 68:193-9, 1956.

[12] DE. Babel, SA. Baughman, "Evaluation of the adult carrier state injuvenile Tinea capitis caused by Trichophyton tonsurans", . J Am Acad Dermatol, 21:1209-12, 1989.

[13] PJ Honig, LR. Smith,"Tinea capitis masquerading asatopic or seborrheic dermatitis", J Pediatr, 94:604-, 1979. 